\title{
Association of the IRF6 rs2235371 and rs861019 Polymorphisms with Non-Syndromic Cleft Lip with or without Cleft Palate in the Yakut Population
}

\author{
Nadezhda I. Pavlova, $\mathrm{PhD}^{1 *}$; Aleksandra T. Diakonova ${ }^{1}$; Vladislav A. Alekseev ${ }^{1}$; \\ Lyubov S. Mironova ${ }^{2,3}$ PGS; Vladimir V. Dodokhov ${ }^{4}, \mathrm{PhD}$; \\ Khariton A. Kurtanov 5 , PhD; Innokenty D. Ushnitsky ${ }^{2}, \mathrm{PhD}, \mathrm{ScD}$ \\ ${ }^{1} Y a k u t$ Science Center of Complex Medical Problems \\ ${ }^{2}$ The North-Eastern Federal University named after M.K. Ammosov \\ ${ }^{3}$ Republic Hospital No. 1 - National Center of Medicine \\ ${ }^{4}$ Arctic State Agrotechnological University \\ ${ }^{5}$ Institute for Biological Problems of Cryolithozone of SB of RAS \\ Yakutsk, the Republic of Sakha (Yakutia), Russia
}

\begin{abstract}
Background: Non-syndromic cleft lip with or without cleft palate (NSCL/P) is one of the most common birth defects. NSCL/P can be broadly divided into cleft lip only (CLO), cleft palate only (CPO), and cleft lip with cleft palate (CLP) based on clinical presentation. The aim of this study was to investigate the relationship between the IRF6 gene polymorphisms and NSCL/P in the Yakut population.

Methods and Results: In 23 OFC patients and 58 unrelated control subjects from the Yakut population, we tested two SNPs (rs2235371 and rs861019) with a minor allele frequency of more than $5 \%$ in the candidate gene IRF6. We found that the SNP marker rs861019 showed significant differences in allele frequencies $(\mathrm{OR}=2.07,95 \% \mathrm{CI}: 1.01-4.23, P=0.04)$ between the NSCL/P patients and the comparison group. Analysis of allele frequencies for rs861019 SNP in subgroups showed that there was a difference in the frequency between CLP and control $(\mathrm{OR}=5.00,95 \% \mathrm{CI}: 1.61-15.53, P=0.11)$; however, this result was not significant.

Genotype analysis showed significant differences in patients from the CLP subgroup in comparison with controls for homozygous (AA compared with $\mathrm{GG})(\mathrm{OR}=9.00,95 \% \mathrm{CI}: 1.03-78.58, P=0.03)$, heterozygous (GA compared with $\mathrm{GG})(\mathrm{OR}=5.50$, 95\% CI: $1.05-28.75, P=0.04$, ), recessive (GG compared with GA + AA) $(\mathrm{OR}=6.67,95 \%$ CI: 1.61-27.58; RR=4.78, 95\% CI: $1.42-$ $16.10, P=0.008$, ) and co-dominant (GG compared with GA, compared with AA) $(P=0.02)$ inheritance models.

Diplotype analysis showed that the NSCL/P group was more likely to have the [CC]-[GG] diplotype than the comparison group. This diplotype carries the risk GG genotype (rs861019) (30.4\%) and does not carry the risk T allele(rs2235371). In the CLP subgroup, two diplotypes ([CT]-[GG] and [CC]-[GG]) were found more often than in the comparison group. Both diplotypes carry the risk GG genotype(rs861019; 33.3\%). In the CPO subgroup, the [CT]-[GG] diplotype was more common. In the CLO subgroup, only two diplotypes ([CC]-[GA] and [CC]-[GG]) were found, both of which were more common than in the comparison group (75\% and $25 \%$ ).. It is likely that these results for the CLO and CPO subgroups were influenced by the small size of both samples. Unlike the NSCL/P and CLP groups, in these samples, diplotypes with the homozygous genotype GG (rs861019) without the homozygous genotype TT (rs2235371) were more common. Diplotypes with a homozygous genotype of the TT risk allele were not found in the studied groups except for the comparison group, where the [TT]-[AA] diplotype was represented by a low frequency $(0.17 \%)$.

Conclusion: The present study provides strong statistical support (for the first time to our knowledge) that genetic variants of the IRF6 rs861019 SNP are associated with NSCL/P in Yakuts.(International Journal of Biomedicine. 2021;11(4):570-575.)
\end{abstract}

Key Words: orofacial cleft •IRF6 gene $・$ rs2235371 • rs861019 • haplotype $・$ diplotype $・$ Yakuts

For citation: Pavlova NI, Diakonova AT, Alekseev VA, Mironova LS Dodokhov VV, Kurtanov KhA, Ushnitsky ID. Association of the IRF6 rs2235371 and rs861019 Polymorphisms with Non-Syndromic Cleft Lip with or without Cleft Palate in the Yakut Population. International Journal of Biomedicine. 2021;11(4):570-575. doi:10.21103/Article11(4)_OA28 


\section{Abbreviations}

CLP, cleft lip with cleft palate; CLO, cleft lip only; CPO, cleft palate only; IRF6, interferon regulatory factor 6; LD, linkage disequilibrium; NSCL/P, non-syndromic cleft lip with or without cleft palate; OFC, orofacial cleft; SNP, single nucleotide polymorphism.

\section{Introduction}

Non-syndromic cleft lip with or without cleft palate (NSCL/P) can be broadly divided into cleft lip only (CLO), cleft palate only (CPO), and cleft lip with cleft palate (CLP) based on clinical presentation. ${ }^{(1)} \mathrm{NSCL} / \mathrm{P}$ is one of the most common birth defects that carries a serious physical and financial burden to affected patients and their families. ${ }^{(2)}$ The overall prevalence of orofacial cleft (OFC) abnormalities is estimated at about 1 in 700 live births, which is almost half of all craniofacial anomalies. ${ }^{(3,4)}$ According to the World Health Organization, the prevalence of $\mathrm{OFC}$ at birth varies worldwide, from 0.34-2.29 per 1000 live births for CLP and 0.13-2.53 per 1000 live births for CPO. ${ }^{(5)}$ The frequency of CLP and CPO can vary greatly from study to study. Prevalence was found to vary by origin, with the highest incidence rates in the Asian population (0.82-4.04 per 1000 live births), intermediate rates among Caucasians (0.9-2.69 per 1000 live births), and the lowest rates among the African population (0.18-1.67 per 1000 live births)..$^{(1,6,7)}$ It was also found that prevalence varied even more by subgroup; for example, one study reported lower OFC rates among residents of the Far East than among Filipinos. ${ }^{(8)}$ The frequency of CLP in Russia is 1:700-1:1000, while in Yakutia (mixed population) it is 1:548 (1.82 per 1000 newborns), and an unfavorable tendency of their increase was noted over the period from 2000-2016. (9,10) $^{(10}$

NSCL/P is a complex malformation that is influenced by both genetic and environmental factors. In early pregnancy, maternal exposure to tobacco smoking, alcohol use, malnutrition, drugs, viral infections, and environmental pollution increases the risk of having children with NSCL/P. ${ }^{(11)}$ Approximately 20 genes are involved in the etiology of NSCL/P, such as IRF6, MSXI, TGF-beta, MTHFR, and FOXE1. ${ }^{(12,13)}$ Among these genes, IRF6, located on the long arm of chromosome 1 (1q32.3-q41), encodes a member of the interferon regulatory transcription factor family. ${ }^{(13)}$ Interferon regulatory factor 6 (IRF6) belongs to a family of nine transcription factors that share a highly conserved helix-turn-helix DNA-binding domain and a less conserved protein-binding domain. Genetic variations in the IRF6 gene were first identified in the etiology of autosomal dominant van der Wood syndrome, which included cleft lip and/or cleft palate and lower lip pits. In addition, the role of the IRF6 gene has also been studied in animal models of NSCL/P. Ingraham et al. ${ }^{(14)}$ reported that the IRF6 knockdown mice have abnormal skin, limb, and craniofacial development. Richardson et al. ${ }^{(15)}$ demonstrated that IRF-6 acts as a key determinant of the keratinocyte proliferation-differentiation switch. Subsequently, these authors further demonstrated that IRF6 plays an important role in the formation and maintenance of the oral peridemis, the spatio-temporal regulation of which is important to ensure adequate palatal adhesion. In 2004,Zucchero et al. ${ }^{(13)}$ investigated 36 SNPs in the IRF6 gene with 8003 individual subjects originating from 10 populations, including Asian, European, and American. One particular SNP (rs2235371) was the first marker in the IRF6 gene that was significantly associated with NSCL/P in Asians and South Americans. Subsequently, similar studies in different populations provided additional evidence that the IRF6 rs2235371 SNP is significantly associated with $\mathrm{NSCL} / \mathrm{P}$. Although the exact functions of the IRF6 gene remain unknown, these findings indicate that the IRF6 gene plays a critical role in NSCL/P. Wang et al. ${ }^{(16)}$ performed the first meta-analysis investigating the relationship between the IRF6 rs2235371 SNP and the risk of NSCL/P. These authors performed subgroup analyses stratified by ethnicity (including Caucasians, Asians, and mixed) and NSCL/P types (CLP and $\mathrm{CPO}$ ), but did not analyze CLO due to the lack of available data. The results showed that genes sensitive to NSCL/P can differ in different ethnic groups and types of NSCL/P. ${ }^{(16)}$ The relationship between the common IRF6 rs2235371 variant and NSCL/P has been widely studied in the world, but the results of these studies are contradictory.

These results are especially controversial in studies with a mixed population, for example, in Brazil, where the results varied depending on the geographic region and the studied ethnicity.(17) Therefore, in order to better understand the association between the IRF6 gene and NSCLP, other polymorphisms are also studied, for example, rs861019. ${ }^{(18,19)}$

The Yakuts, the largest ethnic group of indigenous people of Siberia in Russia with a special life environment, race, customs, and socio-economic status, live mainly in Yakutia (Siberia). The number of Yakuts is about 500,000 people, and their CLP level exceeds the national level.

However, no studies have been done to examine candidate genes associated with NSCL/P among the Yakuts, and we do not know of a single study that has been published in English. Taking into account the genetic heterogeneity of $\mathrm{NSCL} / \mathrm{P}$ in different populations and the negative tendency of an increase in the incidence of NSCL/P in newborns in Yakutia, ${ }^{(10)}$ we conducted a case-control study to find out whether the IRF6 polymorphism affects susceptibility to $\mathrm{NSCL} / \mathrm{P}$ in the Yakuts. This work is a continuation of the previously published work. ${ }^{(20)}$

\section{Materials and Methods}

The experimental part of the work on the genotyping of the IRF6 SNPs (rs2235371, rs861019) was performed in the Department of Molecular Genetics at the Yakutsk Scientific Center for Complex Medical Problems (YSC CMP). For the study, we used DNA samples from the collection of biomaterials of the YSC CMP using the Unique scientific equipment "Genome of Yakutia" (registration no. USU_507512). The sample of the examined persons consisted of 23 children ( 9 girls and 14 boys) of the Yakut ethnicity with congenital OFCs. According to the indications, a cytogenetic examination was performed to exclude chromosomal pathology in this group of children. The comparison group (control) included 58 healthy Yakut volunteers (35 women and $23 \mathrm{men}$ ) who had no history of relatives with congenital 
OFCs. The average age of the patients and the volunteers was $12.09 \pm 2.35$ years and $40.52 \pm 0.19$ years, respectively.

Genomic DNA samples were isolated from the peripheral blood leukocytes using a commercial DNA kit, Excel biotech (Yakutsk, Russia). The study of the IRF6 SNPs (rs2235371, rs861019) was performed by PCR and RFLP analysis.

Primer sequences, conditions for amplification, restriction pattern, restriction enzyme, and the lengths of the restoration fragments are presented in Table 1.

\section{Table 1.}

The primers and restriction enzymes used for detection of IRF6 SNPs using PCR-RFLP methods

\begin{tabular}{|c|c|c|c|c|}
\hline SNP & Primers & $\begin{array}{c}\mathrm{AT} \\
{ }^{\circ} \mathrm{C}\end{array}$ & RE & $\begin{array}{l}\text { RFL, } \\
\text { bp }\end{array}$ \\
\hline \multirow[b]{2}{*}{ rs2235371 } & $\begin{array}{l}\text { F: 5'-ATCAGTCCT } \\
\text { CTGTCCATGACG-3' }\end{array}$ & \multirow{2}{*}{61} & \multirow{2}{*}{ MboI } & \multirow{2}{*}{$\begin{array}{c}\text { CC: } 310 \mathrm{bp}, \\
\text { CT: } 310+222+88 \mathrm{bp} \\
\text { TT: } 222+88 \mathrm{bp}\end{array}$} \\
\hline & $\begin{array}{l}\text { R: 5'-GCATGAGTC } \\
\text { ACAGGGATGAAC-3' }\end{array}$ & & & \\
\hline \multirow{2}{*}{$\begin{array}{l}\text { IRF6 } \\
\text { rs861019 }\end{array}$} & $\begin{array}{l}\text { F: 5'-ATGACACCA } \\
\text { CCATGATGAGGGA-3' }\end{array}$ & \multirow{2}{*}{61} & \multirow{2}{*}{ TfiI } & \multirow{2}{*}{$\begin{array}{c}\text { GG: } 350 \mathrm{bp}, \\
\text { GA: } 350+212+138 \mathrm{bp} \\
\text { AA: } 212+138 \mathrm{bp}\end{array}$} \\
\hline & $\begin{array}{l}\text { R: 5'-CTAGCCATG } \\
\text { CAAAGCTTGTCTC-3' }\end{array}$ & & & \\
\hline
\end{tabular}

$A T$, annealing temperature; $R E$, restriction enzyme; $R F L$ - restriction fragment length; bp, base pair

Genotypes were determined by analyzing the sizes of the resulting fragments by gel electrophoresis on a $4 \%$ agarose gel with ethidium bromide in a standard Tris-acetate buffer at $120 \mathrm{~V}$ for 1 hour. Restriction products were visualized using a UV gel documentation system (Vilber Lourmat, France).

The study was approved by the Ethics Committee of the YSC CMP. Written informed consent was obtained from each research participant (or the participant's parent/guardian).

Statistical analysis was performed using statistical software package SPSS version 17.0 (SPSS Inc, Chicago, IL). Differences in the allele and genotype distribution between the groups were assessed by $\chi 2$ - test or Fisher's exact test, when appropriate. The odds ratio (OR), relative risk (RR) and the corresponding $95 \%$ CI were calculated to estimate the strength of the association.

The following genetic models and test were analyzed: allelic model: D compared with $d$ ( $d$ - the minor allele); genotypic tests - homozygous model (DD compared with dd), heterozygous model (Dd compared with dd); recessive model (dd compared with Dd + DD); dominant model (dd+Dd compared with DD); over-dominant model (DD+dd compared with Dd); co-dominant model (DD compared with Dd, compared with dd). The Haploview 4.2. software (Broad Institute, Cambridge, MA, USA) was use to analyze the Linkage disequilibrium between variants. A probability value of $P<0.05$ was considered statistically significant.

\section{Results and Discussion}

Among OFC patients, the largest number of children ( $n=12,6$ boys and 6 girls) was diagnosed with CLP. CLO and
CPO were diagnosed in 4 children (all boys) and 7 children ( 4 boys and 3 girls), respectively.

In 23 OFC patients and 58 unrelated control subjects from the Yakut population, we tested two SNPs (rs2235371 and rs861019) with a minor allele frequency of more than 5\% in the candidate gene IRF6 (Tables 2 and 3). There were no deviations from HWE for any of the genotyped markers in both groups (Table 2).

Statistical analysis showed that polymorphic variants of the IRF6 gene may be associated with an increased risk of NSCL/P in the Yakut population. Of the two studied SNPs, we found that the SNP marker rs861019 showed significant differences in allele frequencies $(\mathrm{OR}=2.07,95 \% \mathrm{CI} 1.01-4.23$, $\mathrm{P}=0.04$ ) between the NSCL/P patients and the comparison group. Analysis of allele frequencies for rs861019 SNP in subgroups showed that there was a difference in the frequency between CLP and control $(\mathrm{OR}=5.00,95 \% \mathrm{CI}$ : 1.61-15.53, $P=0.11$ ); however, this result was not significant. Genotype analysis showed significant differences in patients from the CLP subgroup in comparison with controls for homozygous (AA compared with $\mathrm{GG})(\mathrm{OR}=9.00,95 \% \mathrm{CI}: 1.03-78.58, P=0.03)$, heterozygous (GA compared with $\mathrm{GG})(\mathrm{OR}=5.50,95 \% \mathrm{CI}$ : 1.05-28.75, $P=0.04)$, recessive (GG compared with $\mathrm{GA}+\mathrm{AA}$ ) $(\mathrm{OR}=6.67,95 \% \mathrm{CI}: 1.61-27.58 ; \mathrm{RR}=4.78,95 \% \mathrm{CI}: 1.42-16.10$, $P=0.008)$ and co-dominant (GG compared with GA, compared with AA) $(P=0.02)$ inheritance models.

To show the genetic relationship between the tested SNPs, paired LD analysis and haplotype estimation were performed for the studied SNPs. Haplotypes were constructed using the data on the genotypes we obtained in this work. LD patterns for combinations of 2 SNPs are shown in Table 4 and Figure 1 There was very weak LD between two SNPs of the IRF6 gene (Table 3 and Fig. 1) in almost all groups, except for the CPO subgroup, where a weak LD was observed $(\mathrm{D} '=1$ with LOD = 2). The frequency distribution of the IRF6 gene haplotypes for two SNPs (rs2235371 and rs861019) is shown in Table 3.

There are four possible haplotypes (CG, CA, TA, and TG) for the rs2235371 and rs861019 SNPs. All four haplotypes were found in the NSCL/P group and ComG. The fact that the TG and TA haplotypes were not found in the CPO and CPO subgroups probably indicates the small size of these subgroups. The frequency of 2-marker combined haplotypes did not show significant differences from the comparison group.

The distribution of diplotype frequencies for two SNPs (rs2235371 and rs861019) of the IRF6 gene, based on all detected variants, is presented in Table 4. Seven diplotypes out of nine possible variants were found. The NSCL/P group was more likely to have the $[\mathrm{CC}]-[\mathrm{GG}]$ diplotype than the comparison group. This diplotype carries the risk GG genotype (rs861019) (30.4\%) and does not carry the risk T allele(rs2235371). In the CLP subgroup, two diplotypes ([CT]-[GG] and [CC]-[GG]) were found more often than in the comparison group. Both diplotypes carry the risk GG genotype(rs861019; 33.3\%). In the CPO subgroup, the [CT]-[GG] diplotype was more common. In the CLO subgroup, only two diplotypes ([CC]-[GA] and [CC][GG]) were found, both of which were more common than in the comparison group (75\% and 25\%). 
Table 2.

Association of the IRF6 rs2235371 and rs861019 SNPs with the development of NSCL/P, CLP, CLO, CPO

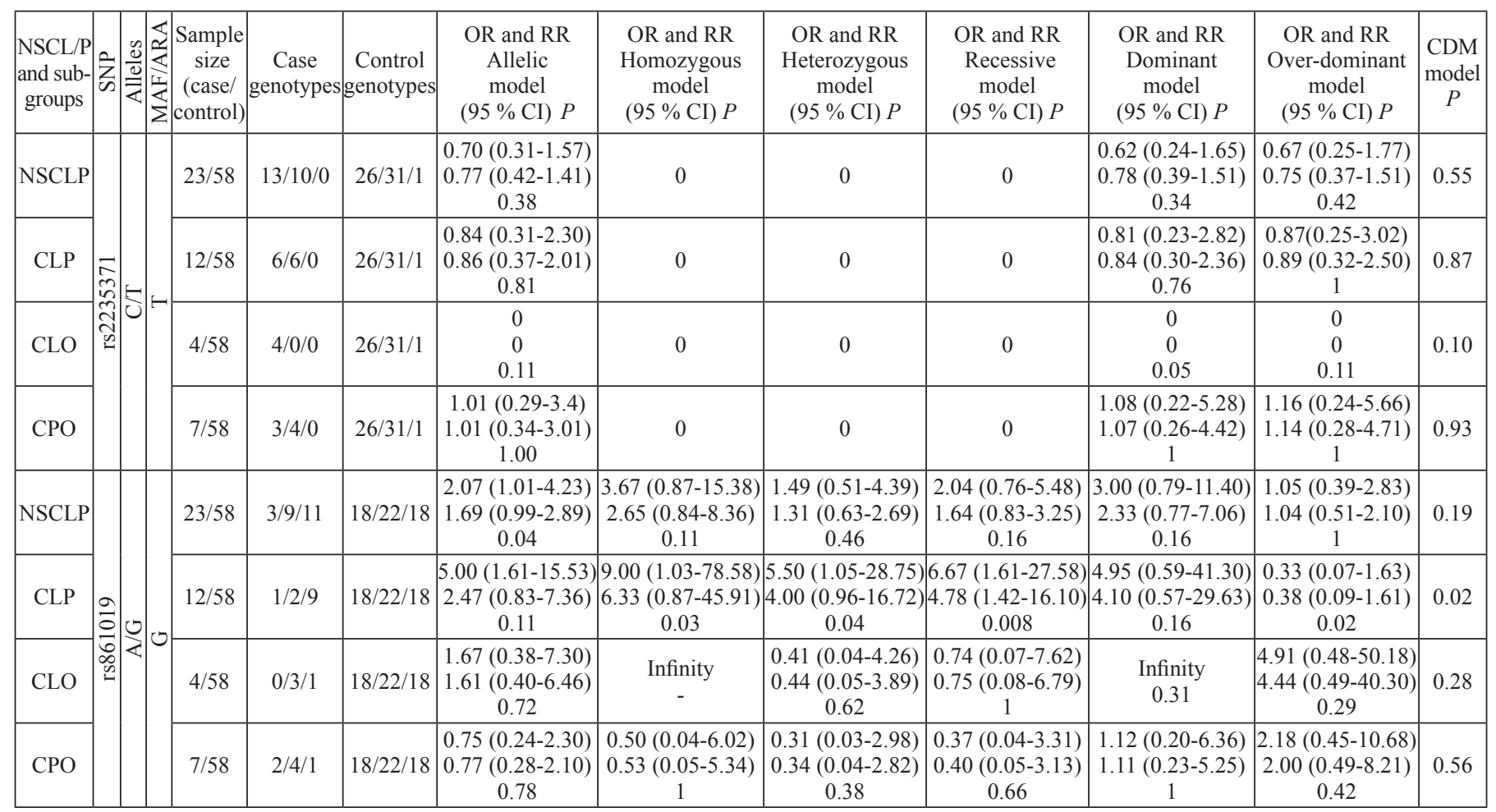

The order of genotypes: DD/Dd/dd (d is the minor allele); Allelic model: D compared with d; Homozygote model: DD compared with dd; Heterozygote model: Dd compared with dd; Recessive model: $d d$ compared with Dd+DD; Dominant model: $d d+D d$ compared with DD; Over-dominant model: DD+dd compared with Dd; Co-dominant model: DD compared with Dd, compared with dd; P: HWE P-value. ARA, associated risk allele; CDM, Co-dominant model

Table 3.

The frequency distribution of IRF6 gene haplotypes for two SNPS (rs2235371 and rs861019) of the IRF6 gene in Yakuts with NSCL/P and OFC subgroups

\begin{tabular}{|c|c|c|c|c|c|c|c|}
\hline \multirow{2}{*}{$\begin{array}{c}\text { NSCL/P } \\
\text { and } \\
\text { subgroups }\end{array}$} & \multicolumn{4}{|c|}{ Haplotype frequency } & \multicolumn{3}{|c|}{ Linkage disequilibrium (LD) } \\
\cline { 2 - 8 } & T-G & C-G & T-A & C-A & D' & LOD & $\mathrm{r}^{2}$ \\
\hline $\begin{array}{c}\text { NSCLP } \\
(\mathrm{n}=23)\end{array}$ & 0.123 & 0.377 & 0.161 & 0.339 & 0.115 & 0.02 & 0.008 \\
\hline $\begin{array}{c}\text { Control } \\
(\mathrm{n}=58)\end{array}$ & 0.130 & 0.544 & 0.088 & 0.238 & 0.134 & 0.08 & 0.007 \\
\hline $\begin{array}{c}\text { CLP } \\
(\mathrm{n}=12)\end{array}$ & 0.178 & 0.656 & 0.072 & 0.094 & 0.247 & 0.12 & 0.036 \\
\hline $\begin{array}{c}\text { CPO } \\
(\mathrm{n}=7)\end{array}$ & 0 & 0.625 & 0 & 0.375 & 1 & 0.77 & 0.533 \\
\hline $\begin{array}{c}\text { CLO } \\
(\mathrm{n}=4)\end{array}$ & 0.286 & 0.143 & 0 & 0.571 & 0 & 0 & 0 \\
\hline
\end{tabular}

$D^{\prime}$, the coefficient of $L D ; r^{2}$, an alternative to $D^{\prime}$ is the correlation coefficient between pairs of loci, usually expressed as its square.
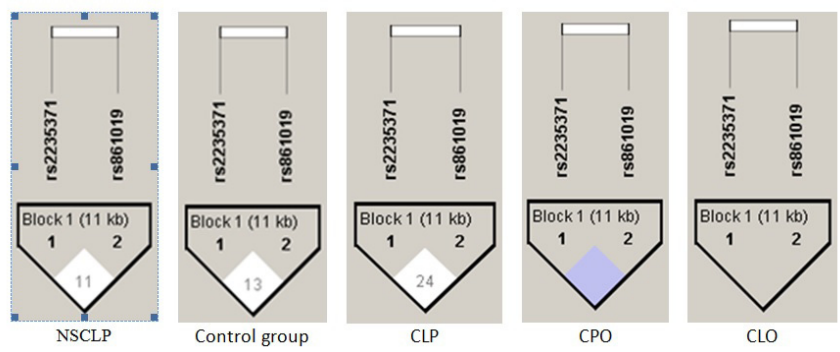

Fig. 1. LD between the IRF6 (rs2235371, rs861019) SNPs

The color scheme shows the strength of adhesion between SNPS: white squares - a poor link $\left(D^{\prime}<1, L O D<2\right)$; grey squares a strong kink $\left(D^{\prime}=1\right.$ with $\left.L O D=2\right) ; k b$, kilobase.

It is likely that these results for the CLO and CPO subgroups were influenced by the small size of both samples. Unlike the NSCL/P and CLP groups, in these samples, diplotypes with the homozygous genotype GG (rs861019) without the homozygous genotype TT (rs2235371) were more common. Diplotypes with a homozygous genotype of the TT risk allele were not found in the studied groups except for the comparison group, where the [TT]$[\mathrm{AA}]$ diplotype was represented by a low frequency $(0.17 \%)$.

\section{Table 4.}

Distribution of diplotypes for two SNPs (rs2235371 and rs861019) of the IRF6 gene in NSCLP patients and the comparison group

\begin{tabular}{|c|c|c|c|c|c|c|c|c|}
\hline \multirow[b]{2}{*}{ № } & \multicolumn{2}{|c|}{ Genotype / SNP } & \multirow[b]{2}{*}{ Diplotype } & \multicolumn{5}{|c|}{ Diplotype frequency } \\
\hline & rs2235371 & rs861019 & & $\begin{array}{l}\mathrm{NSCL} / \mathrm{P} \\
(\mathrm{n}=23)\end{array}$ & $\begin{array}{l}\text { Control } \\
(\mathrm{n}=58)\end{array}$ & $\begin{array}{c}\text { CLP } \\
(\mathrm{n}=12)\end{array}$ & $\begin{array}{l}\mathrm{CPO} \\
(\mathrm{n}=7)\end{array}$ & $\begin{array}{l}\text { CLO } \\
(\mathrm{n}=4)\end{array}$ \\
\hline 1 & TT & GG & {$[\mathrm{TT}]-[\mathrm{GG}]$} & 0.000 & 0.000 & 0.000 & 0.000 & 0.000 \\
\hline 2 & $\mathrm{CT}$ & GG & {$[\mathrm{CT}]-[\mathrm{GG}]$} & 0.174 & 0.155 & 0.333 & 0.077 & 0.000 \\
\hline 3 & TT & GA & {$[\mathrm{TT}]-[\mathrm{GA}]$} & 0.000 & 0.000 & 0.000 & 0.000 & 0.000 \\
\hline 4 & $\mathrm{CT}$ & GA & {$[\mathrm{CT}]-[\mathrm{GG}]$} & 0.217 & 0.224 & 0.111 & 0.308 & 0.000 \\
\hline 5 & TT & AA & {$[\mathrm{TT}]-[\mathrm{AA}]$} & 0.000 & 0.017 & 0.000 & 0.000 & 0.000 \\
\hline 6 & $\mathrm{CC}$ & AA & {$[\mathrm{CC}]-[\mathrm{AA}]$} & 0.087 & 0.138 & 0.056 & 0.154 & 0.000 \\
\hline 7 & $\mathrm{CC}$ & GA & {$[\mathrm{CC}]-[\mathrm{GA}]$} & 0.174 & 0.155 & 0.111 & 0.231 & 0.750 \\
\hline 8 & $\mathrm{CC}$ & GG & {$[\mathrm{CC}]-[\mathrm{GG}]$} & 0.304 & 0.155 & 0.333 & 0.077 & 0.250 \\
\hline 9 & $\mathrm{CT}$ & AA & {$[\mathrm{CT}]-[\mathrm{AA}]$} & 0.043 & 0.155 & 0.056 & 0.154 & 0.000 \\
\hline
\end{tabular}


IRF6 belongs to a family of nine transcription factors that play a key role in the formation and maintenance of the oral peridemis and palatine shelves. Knockdown of the IRF6 gene in mice showed an abnormal multilayer development of the epidermis, skin, limbs, and craniofacial regions, and gene expression analysis showed that the primary defect was in the proliferation-differentiation switch of keratinocytes. Since Zucchero et al. ${ }^{(13)}$ found a significant association between the IRF6 gene and non-syndromic clefts in several populations from Asia, Europe, and North and South America, a number of replication studies have been conducted with different populations and ethnic groups. Notably, one SNP (rs2235371) that changed the conserved amino acid valine to isoleucine at codon 274 in the SMIR-binding domain was significantly associated with cleft lip and palate, especially in Asians and South Americans. We assessed 2 SNPs of the IRF6 gene in 81 subjects and found evidence of an association between these SNPs and NSCL/P in the Yakut population, further supporting previous findings that the IRF6 gene is involved in the pathogenesis of NSCL/P. Patients with NSCL/P had a significantly higher frequency of the $G$ allele than the comparison group, with a relatively high odds ratio $(\mathrm{OR}=2.07$, 95\% CI: 1.01-4.23, $P=0.04)$. This indicates that patients with the rs $861019 \mathrm{G}$ allele are predisposed to a 2.07 -fold increased risk of developing this anomaly. As far as we know, the relationship between the IRF6 common variant rs861019 and non-syndromic cleft mouth has been extensively studied around the world. However, to date, the results remain controversial. Inconsistent results may arise due to different ethnic origins, differences in environment, anthropological diversity, different research methods, and complex genetic etiology of the disease. It is generally accepted that any SNP can have only a moderate effect, and combined variants within a gene can provide a more complete assessment in association studies. When LD between markers is weak, haplotype and diplotype analyses have advantages over SNP alone. The results of our diplotype analysis are consistent with the results of the genotypic analysis for rs861019, that is, that the $G$ allele is a risk factor for NSCL/P. We also suspect that patients carrying the $[\mathrm{CC}]-[\mathrm{GG}]$ diplotype are at a higher risk of developing NSCL/P. Based on these results, we hypothesized that diplotype analysis might be useful to assess the relationship between haplotypes and NSCL/P. Patients with more risk alleles are more likely to develop NSCL/P than patients with fewer or no alleles.

One of the main limitations of this study is the relatively small sample size; some of the minor genotypes are number fewer than five. Therefore, our study was probably not powerful enough to detect a mild to moderate association between SNPs and clefts. The most widely studied marker rs2235371 in the IRF6 gene, which showed a significant association with an increased risk of NSCL/P in Europeans and Asians, in our study did not show an association with NSCL/P.

\section{Conclusion}

In the present case-control study, there were no significant associations between the IRF6 rs2235371 SNP and
NSCL/P. The present study provides strong statistical support (for the first time to our knowledge) that genetic variants of the IRF6 rs861019 SNP are associated with NSCL/P in Yakuts. Despite the discovery of an association between the rs861019 SNP and the risk of NSCL/P in our patients, more studies are required in similar populations, but with larger sample sizes, to further explore potential associations with different NSCL/P sub-phenotypes and to determine the structure of IRF6 genetic variants in populations, for example, in Yakutia.

\section{Sources of Funding}

The research was carried out within the framework of the project "Physiological and biochemical mechanisms of adaptation of plants, animals, humans to the conditions of the Arctic/Subarctic and the development of biological products based on natural northern raw materials that increase the efficiency of the adaptation process and the level of human health in extreme environmental conditions" (No. 0297-20210025 registration number AAAA-A21-121012190035-9) and the R\&D "Study of the genetic structure and burden of hereditary pathology of populations of the Republic of Sakha (Yakutia)" (No. USU_507512).

\section{Competing Interests} interests.

\section{References}

1. Jugessur A, Rahimov F, Lie RT, Wilcox AJ, Gjessing HK, Nilsen RM, Nguyen TT, Murray JC. Genetic variants in IRF6 and the risk of facial clefts: single-marker and haplotypebased analyses in a population-based case-control study of facial clefts in Norway. Genet Epidemiol. 2008 Jul;32(5):41324. doi: 10.1002/gepi.20314.

2. Wehby GL, Cassell CH. The impact of orofacial clefts on quality of life and healthcare use and costs. Oral Dis. 2010 Jan;16(1):3-10. doi: 10.1111/j.1601-0825.2009.01588.x.

3. Mossey PA, Modell B. Epidemiology of oral clefts 2012: an international perspective. Front Oral Biol. 2012;16:1-18. doi: $10.1159 / 000337464$.

4. Gorlin RJ, Cohen MM, Hennekam RCM. Syndromes of the Head and Neck. New York: Oxford University Press; 2001. 5. Mossey PA, Castilla EE. Global Registry and Database on Craniofacial Anomalies: Report of a WHO Registry Meeting on Craniofacial Anomalies. Geneva, Switzerland: World Health Organization; 2001.

6. Allam E, Windsor L, Stone C. Cleft lip and palate: etiology, epidemiology, preventive and intervention strategies. Anat Physiol. 2014;4: 940-2161. doi:10.4172/2161-0940.1000150 7. Ahmed MK, Bui AH, Taioli E. Epidemiology of Cleft Lip and Palate. Designing Strategies for Cleft Lip and Palate Care. In TechOpen. 2017;22. doi: 10.5772/67165

*Corresponding author: Nadezhda I. Pavlova, PhD. Yakut Science Center of Complex Medical Problems. Yakutsk, the Republic of Sakha (Yakutia), Russia. E-mail: solnishko_84@inbox.ru 
8. Forrester MB, Merz RD. Descriptive epidemiology of oral clefts in a multiethnic population, Hawaii, 1986-2000. Cleft Palate Craniofac J. 2004 Nov;41(6):622-8. doi: 10.1597/03-089.1.

9. Abdurahmonov AZ. [Congenital cleft lip and palate in children from Tajikistan in 2009-2019]. Aspirantskiy Vestnik Povolzhiya. 2020;20(1-2):75-79. doi: 10.17816/20722354.2020.20.1.75-79. [Article in Russian].

10. Ushnitsky ID, Mironova LS, Gogolev II, Davydova MM. Clinical and genetic aspects of congenital lip and palate clefts in children of Yakutia. Yakut Medical Journal. 2018; 1(61). doi: 10.25789/YMJ.2018.61.06

11. Dixon MJ, Marazita ML, Beaty TH, Murray JC. Cleft lip and palate: understanding genetic and environmental influences. Nat Rev Genet. 2011 Mar;12(3):167-78. doi: $10.1038 /$ nrg2933.

12. Jagomägi T, Nikopensius T, Krjutskov K, Tammekivi V, Viltrop T, Saag M, Metspalu A. MTHFR and MSX1 contribute to the risk of nonsyndromic cleft lip/palate. Eur J Oral Sci. 2010 Jun;118(3):213-20. doi: 10.1111/j.1600-0722.2010.00729.x.

13. Zucchero TM, Cooper ME, Maher BS, Daack-Hirsch S, Nepomuceno B, Ribeiro L, et al. Interferon regulatory factor 6 (IRF6) gene variants and the risk of isolated cleft lip or palate. N Engl J Med. 2004 Aug 19;351(8):769-80. doi: 10.1056/ NEJMoa032909.

14. Ingraham CR, Kinoshita A, Kondo S, Yang B, Sajan S, Trout KJ, Malik MI, Dunnwald M, Goudy SL, Lovett M, Murray JC, Schutte BC. Abnormal skin, limb and craniofacial morphogenesis in mice deficient for interferon regulatory factor 6 (Irf6). Nat Genet. 2006 Nov;38(11):1335-40. doi: $10.1038 /$ ng 1903.

15. Richardson RJ, Dixon J, Malhotra S, Hardman MJ,
Knowles L, Boot-Handford RP, Shore P, Whitmarsh A, Dixon MJ. Irf6 is a key determinant of the keratinocyte proliferationdifferentiation switch. Nat Genet. 2006 Nov;38(11):1329-34. doi: $10.1038 / \mathrm{ng} 1894$.

16. Wang M, Pan Y, Zhang Z, Wang L. Three polymorphisms in IRF6 and 8q24 are associated with nonsyndromic cleft lip with or without cleft palate: evidence from 20 studies. Am J Med Genet A. 2012 Dec;158A(12):3080-6. doi: 10.1002/ ajmg.a.35634.

17. de Souza LT, Kowalski TW, Ferrari J, Monlléo IL, Ribeiro EM, de Souza J, Fett-Conte AC, de Araujo TK, Gil-da-SilvaLopes VL, Ribeiro-Dos-Santos ÂK, dos Santos SE, Félix TM. Study of IRF6 and 8q24 region in non-syndromic oral clefts in the Brazilian population. Oral Dis. 2016 Apr;22(3):241-5. doi: 10.1111/odi.12432.

18. Rafighdoost H, Hashemi M, Danesh H, Bizhani F, Bahari G, Taheri M. Association of single nucleotide polymorphisms in AXIN2, BMP4, and IRF6 with Non-Syndromic Cleft Lip with or without Cleft Palate in a sample of the southeast Iranian population. J Appl Oral Sci. 2017 Nov-Dec;25(6):650-656. doi: 10.1590/1678-7757-2017-0191.

19. Pegelow M, Peyrard-Janvid M, Zucchelli M, Fransson I, Larson O, Kere J, Larsson C, Karsten A. Familial nonsyndromic cleft lip and palate--analysis of the IRF6 gene and clinical phenotypes. Eur J Orthod. 2008 Apr;30(2):169-75. doi: 10.1093/ejo/cjm097.

20. Pavlova NI, Kurtanov KhA, Diakonova AT, Mironova LS, Solovyeva NA, Borisova YP, et al. Genetic Predictors for the Development of Congenital Orofacial Clefts. International Journal of Biomedicine. 2020;10(1):50-53. doi: 10.21103/ Article10(1)_OA7 